\title{
Achieving Strategic Growth in Microenterprises through Information Technology: UK Micro Enterprise Case Study
}

\author{
S. Shah ${ }^{1}$, M. Long ${ }^{1}$, E. Naghi Ganji ${ }^{1}$, \\ ${ }^{1}$ Department of Applied Engineering and Management, FES, University of Greenwich, Chatham, United Kingdom \\ (S.shah@gre.ac.uk, M.long@gre.ac.uk, E.naghiganji@gre.ac.uk)
}

\begin{abstract}
Technology is a powerful tool that aims to assist efficient and effective use of resources within businesses. The paper examines to provide an understanding on the use of Information Technology tools and its influence on a UK microenterprise. It aims to explore further understanding on the strategic growth of any microenterprise firms. Through research findings and that of the analysis, it develops reasons and factors towards achieving greater time efficient practices with the use of information technology tools to achieve strategic growth for the business in the challenging economic markets. The paper presents the preliminary case study of UK microenterprise through observational findings examining general business processes, the challenges and drawbacks within the working environments and that of the use of technological tools. The findings of this case study will further enable the researchers to develop a novel framework to assist and enable any microenterprise in achieving overall strategic growth.
\end{abstract}

Keywords - Information technology (IT), microenterprises, small and medium enterprises (SME), strategic growth.

\section{INTRODUCTION}

Information Technology is currently seeing the biggest transformation within global business and technology forums. With the change in direction from the traditional application and facilitation use, it has now widespread application enabling its use towards more of empowering businesses to provide more effective, responsive and valuable service to their consumers. It has also enabled many of organisations to achieve more competitive edge in addressing complex issues and providing solutions based upon the needs and wants of the user. It has provided the transformation of converting the daily tasks and activities within the administration aspects of the business more efficient, thus enabling the proper use of technical and managerial skills within the company. It also allows the proper use of time, achieve cost effective measures and be able to expand the future prospects for the businesses. Many of the larger corporations have the capacity towards investing heavily in technology, allowing them to adopt more innovative and entrepreneurial ways towards being more process incentive, thus achieving better success and profits for the organisation [1]. Many a times these large corporations and businesses have the capacity in terms of manpower and technical skills that allows them to address any problems by capturing the requirements and developing appropriate solutions towards implementation. However, for much of the microenterprises within the UK, their ability to develop better ways to conduct their daily operations with the prospects of future growth could be limited and at times difficulty to achieve due to the limitation of resources and technical expertise [2]. The paper aims to focus towards addressing some of the key research questions related the aspect focusing towards the Microenterprises within UK and their ability to adopt and implement more technology related applications within their business environments. The researchers through this papers intends to identify reasons towards microenterprises having greater awareness within the business communities compared to that of small and medium enterprises (SMEs). It also aims to examine if many of these microenterprises aspires to grow and convert towards becoming larger or SME compared to that of those who prefer to remain micro through their operations. And finally, the paper also will address and examine the current perceptions of Information Technology and its impact to microenterprises that are aiming to pursue and achieve strategic growth within their business transformation strategies.

\section{LITERATURE STUDY}

The focus of this paper is to examine the use of information technology and its influences towards UK microenterprises aiming to achieve overall strategic growth within the market space. With the use of current literature surrounding the topic it will enable the researchers to understand the background of microenterprises within the UK economy and the challenges faced by these firms within their environments. The researchers also aim to analyse the linkages between microenterprises and that of their technology adoption. The authors also aims to examine the key challenges and the decision making process towards technology adoption and how this affects the strategic direction for the firm. There are very varied interpretations across the research community and that of professional and organisational institutions towards defining a "microenterprise". The main reason for the differentiation lies within categorization of businesses on the basis of their size which is governed by many of the governmental policies within the county. For instance, the EU defines SMEs through the size and those that are able to benefit from funding programmes and that of various policies that surrounds the SME. Contrary to this, the same 
regulations restricts smaller firms to benefit from those incentives as they may be "exceeding their defined boundaries and hence do not necessarily benefit from those support policies that are intended for SMEs" [3]. However, it has started to become more common that microenterprises are not always regarded within their own and many a times combined in the terminology of "small enterprise" [4, 5]. Similarly, the Department for Business Innovation and Skills for the UK defines microenterprise in their 2009 report [6] do not even include the definition or refer to the term "micro" at all under their Business Population estimates for the UK and regions, 2012". Instead, their definition refers to "small businesses" as having between 0-49 employees [6]. Studies have highlighted that there is no definite agreements or conclusions within the literature about the key factors towards success while suggesting that results towards this varies across different studies conducted within different sectors [7]. Similarly, larger organisations are much driven towards growth and are able to exploit markets within the best of their abilities through the maximization of their profits, whereas, microenterprises are very much diverse in nature and hence not all of these firms strive for growth but more towards sustaining themselves in terms of the competition and that of continued economic challenges faced within their environments [8]. The FSB report suggests that there are certain "growth oriented microenterprises" which accounted towards $57 \%$ of UK microenterprises that were deemed to grow in next two years. Similar studies also suggested the following list of characteristics towards achieving a positive relation towards microenterprises growth $[9,15]$ :

- The level of educational understanding of the entrepreneur;

- Entrepreneurial intensity of the firm;

- Networking informally with the key customers and suppliers of the business;

- Business partnering activities and opportunities;

- Innovation in product and service;

- Adoption and utilization of E-commerce technologies and tools;

- Managerial focus;

- Key focus towards local and regional markets;

- Size and age of the firm;

This rapid response in terms of the development of technological advances has resulted in a diverse range of information technology tools that are more viable and considered as cost effective solutions towards many of the commercial needs within the firms. With more faster and quicker responsive abilities and processing power, and the key abilities towards storing larger amounts of data that can be retrieved more responsively in shorter timescales with the use of simple and efficient analytical and processing tools [11]. There is also lack of research towards SMEs and their understanding towards the key factors where the decision makers and firm executives are influenced towards their decisions in terms of investment and adoption of technology within the firms. This also restricts their growth prospects in future and their ability to be competitive within the market. There is also a lack of understanding on the factors that separates or distinguishes the microenterprises compared to that of their larger business rivals [12].

Existing studies have lighted towards that not every microenterprise are driven towards succeeding by transforming themselves in terms of growth. This is however, very much dependent upon the values, beliefs and the preferences the owners of microenterprises share regarding strategic growth for their firms. The reviews also highlights towards the importance of this aspect and that of their perceptions towards information technology. It is evident that technology has enabled many microenterprises to compete more with that of their larger business counterparts more on personalized levels due to their adaptability and flexibility. However, there is no clarity towards the key factors that can further strengthen this points. It has been seen through earlier research that growth and success of any firm should be defined qualitatively, based upon the firms vision, values, lifestyles, goals and the quality of services provided by the firm to all its partners, including that of consumers [5]. There is strong evidence that suggests that in order for the microenterprises to improve their internal processes and fulfil their administrative tasks more effectively and efficiently with the use of IT, it will further allow the firms to utilise more time towards engaging with their customer base, invest time towards exploring new markets and products, and be able to utilise their resources more towards the areas which will allow their firms to achieve the strategic growth required. There is also empirical evidence that suggests the preferred way towards increasing the profitability is not through cost saving and cutting measures, but through improvement in revenue streams by achieving higher levels of service efficiency, quality and satisfaction levels within the firm [11]. It is also evident that through lack of resources, government support and that of the challenges and barriers facing, it is seen that IT has the potential towards supporting microenterprises to fulfil their goals and be competitive.

It has long been seen that technology has always been looked upon as the key aspect to achieve productivity within many sectors including production and manufacturing based industries. More recently, this has shifted due to the change in technological advancements allowing businesses to have substantial growth and leverage to achieve higher competitive advantages compared to that of their counterparts. The innovation in technology within the services provision is much based towards the adoption and implementation of ICT and hence much associated towards higher levels of performance and productivity within businesses. For many businesses that operate in more competitive markets, there is always an added edge required to remain and sustain the competitive advantage. And this radical and rapid moving changes within the technology then challenges many businesses to have continuous improvement towards adoption and 
implementation of new technological applications and innovative practices within their business environments to achieve higher competitive advantage and improve their business propositions [14].

\section{METHODOLOGY}

The research presents the use of combined qualitative and quantitative research methods. The paper discussed the first method of that of an observational case study that involved observations conducted on individual participants and that of business processes within real microenterprise environment. The method allowed the researchers to examine real case examples towards the challenges and reasons of influence of information technology tools and its adoption within a microenterprise environment. This also allowed the researchers to further validate the findings to that of the literature examined within the study. The study was conducted on a real microenterprise named PMM to enable the researchers to observe and examine the technology implications and how employees within the company were utilizing the current applications on day to day operations of the business.

\section{CASE STUDY ANALYSIS}

The study presents the findings from the case study and that of an observational study conducted for an examined microenterprise. The findings consist of observations of normal office presence and that of the key discussions of importance on strategic levels within the enterprise. Key focus was towards the levels of interests and attention towards employee interactions on the use of information technology applications and tools towards their administrative tasks and that of administrative responsibilities. There were certain challenges and that of drawbacks surrounding these observations which required to be investigated further. The enterprise PMM (name changed for this study) is a microenterprise company based in UK that offers project management services to publishers, academic institutions and that of service based organisations including the healthcare sector. Collecting information from transcripts through to final publications, the company PMM manages all aspects of publishing, including managing the authors and virtual teams from project start to end. The management and that of the administrative teams do not always have personal contact with the clients and much of the work requires completion through online platforms.

\section{RESULTS}

Through the analysis of the qualitative data there were a few questions that began to show common occurrences across participants thus enabling to demonstrate quantifiable visual representations of the data obtained.
This therefore indicates that there could be similar challenges and experiences expressed across a general consensus of a microenterprise.

\section{A. Awareness of the Term "Microenterprises"}

The results of the survey show that a staggering $88 \%$ of respondents do not use the term "micro" to define an enterprise with fewer than nine employees. Interestingly only a third of Current Directors of microenterprises would consider their business as "micro" too. The results support current literature on the lack of defining microenterprises in their own right despite international recognition of terminology [4], [5]. This does not help the argument for the Government to do more to differentiate microbusinesses from the term SME to enhance support and funding [2].

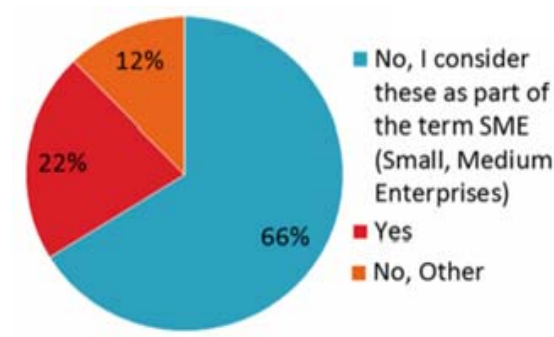

Fig. 1. Survey results towards defining company employing fewer than 10 people.

However, one participant made an interesting point that they "do not determine size of the company by employees, a nine employee firm can manage a multimillion-pound company online", supporting the reason why revenues are also considered and why Governments should do more to recognise the majority that fit within the recognised criteria that lack the financial means to get the help they are entitled towards [5].

\section{B. Strategic Growth of Microenterprises}

Continuing from the definition of a microenterprise it is assumed that participants mentioned terms such as "earlystage" and "looking to expand" which highlights [13] identification of a misconception that all microenterprises aim to grow in size. This is conversely supported by one of the survey questions showing that only $44 \%$ of businesses' current strategy is to grow (figure 2).

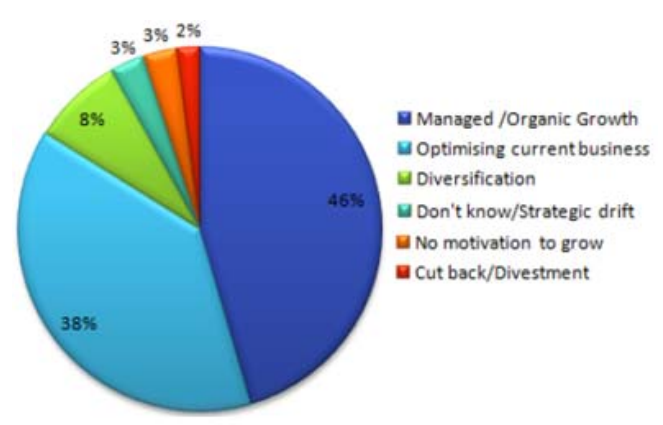

Fig. 2. Results showing microenterprises current strategy 
However, the $38 \%$ of businesses are seeking to optimise their current business does not necessarily prove that the business will look to grow in the future, identified by the observational study of PMM. From Survey participants however stated their reasoning and growth patterns are justified by their employee plans.

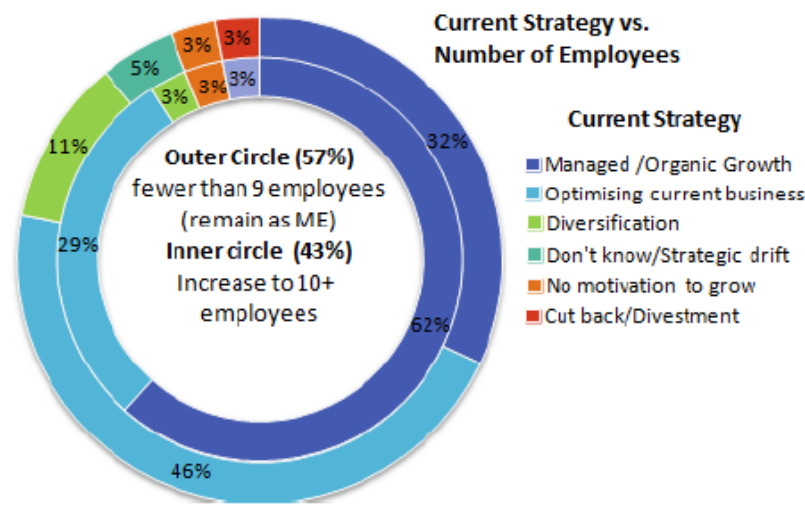

Fig. 3. Representation of survey results of current strategies vs. that of number of employees

As seen in figure 3 , there is a staggering difference between strategies and employment plans, business are almost twice as likely to adopt a growth strategy if they are set to increase employees above 10 whilst almost half of all business that want to remain a microenterprise are seeking optimizing business strategies (46\%).

\section{Current Perceptions of IT Impacts}

To address the research question towards the perceptions of Information Technology and its impact towards pursuing strategic growth; the vast majority (78\%) of Microenterprises consider the use of IT as a critical success factor to their business. The figure 4 presents what proportion of participants see IT as a critical success factor in two ways: that it is integral to the business, secondly the vast amount of benefits to the business Information Technology offers.

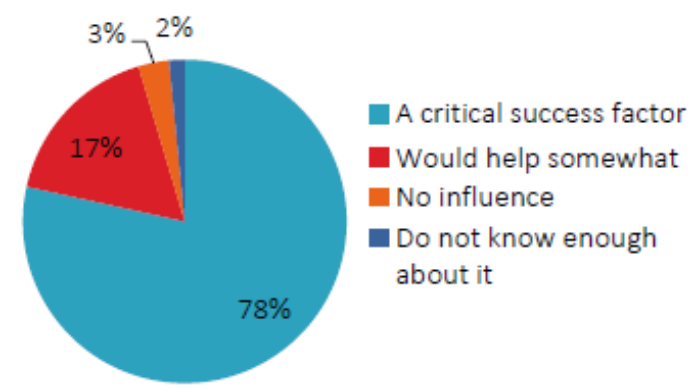

Fig. 4. Survey results of IT use within microenterprise strategies

The benefits addressed, largely ease of data storage and buzzwords related to efficiency, future opportunities, IT has a key part in influencing microenterprise strategic growth. Of the $11 \%$ that consider IT would help somewhat claim it is not critical to the business but helps in three ways: a) Improve communications both internally and externally;

b) Supports increasing efficiency of its processes,

c) Increase sales with online presence;

\section{DISCUSSION}

With a firm understanding that Microenterprises need to have a greater awareness in society, further research or a proposal plan could be developed to attempt to successfully promote microenterprises on their own right with the shifting momentum of improving financial backing and support from the UK Government. Interestingly $25 \%$ of respondents that are currently directors said they would be happy for a group of University students to find ways of optimizing their business through the use of IT. Future research would enable continued research into the influence of IT on microenterprises particularly in aid of optimising strategic growth and overcoming the research problem further.

With the survey conducted to online audience for this research due to the limited market exposure and time constraints, immediately this has opened an opportunity of research into locating and identifying microenterprises that do not use the internet and to find out why they are choosing not to use information technologies in their business. Close observations and monitoring could lead to interesting results if there is an implementation and adoption of IT within the microenterprises. The scope of the survey was to address a generic standpoint on microenterprises and personal viewpoints and perceptions, there is an opportunity to identify if the age of the microenterprise has influence on the strategic direction and use of IT, but also the age of the Director with the possible hypothesis that IT has a greater critical influence on the microenterprise led by a younger director due to their greater exposure and curiosity of the latest technologies. This study also investigated that the grouping of given industries of participants' current job role was misaligned with over a quarter opting for "other" commenting on more specialised industries thus calling for future research to perhaps narrow and focus on the effects of IT on strategic growth in microenterprises in particular industries and/or to look into how microenterprises characterise themselves in the business world. Inversely, a wider study could be conducted to identify a broader and more accurate influence of IT on microenterprises aiming to pursue strategic growth from a generic perspective, this could help further studies in addressing microenterprise importance in the British industry and highlighting the importance to the UK government to do more to break down the term SME.

With the Proposed Solution Framework developed, major emphasis should be placed further investigating how this framework could be expanded further. This could be a development of a full scale solution, research could focus on further implementation to see its effectiveness and capabilities within a microenterprise, perhaps identifying the consistency or differing effects it has to play on 
differing microenterprises and industries. There is opportunity for further enhancements as the literature highlighted tools that combined could enhance or improve the function of the framework in achieving better strategic growth in microenterprises. From highlighting just a few solutions to highlight the influence of IT on microenterprises pursuing strategic growth, further research and development of cost effective management solutions would be beneficial. There are a realm of opportunities and future directions to further enhance the findings from this thesis. The author aims to develop skills and knowledge within the world of business and information technology through the eyes of consulting and helping businesses to optimise, reduce costs or increase business offering.

\section{CONCLUSION}

The study aimed at addressing the need to develop a thorough understanding for microenterprises in achieving greater levels of awareness within the society than that of differentiating towards small and medium enterprises (SMEs). The paper aims to evaluate if microenterprises are driven towards growth or remain "micro" for strategic and financial reasons. This paper outlines the assessment of information technology tools and its influences on microenterprises (MEs). It can be summarised that microenterprises treat IT critically but lack the time, money and know-how to fully optimise their business ready for strategic growth, regardless whether they want to achieve grow in size or merely to pursue personal objectives. Despite little recognition to differentiate microenterprises generally in society, with an established definition and considerable differing characteristics of their slightly larger counterparts 'Small enterprises', more emphasis should be provided to promote microenterprises as an individual entity. Microenterprises prefers to add value while increasing business through greater IT influence towards their strategic growth in turn increasing their profits.

A number of barriers identified that are of greater importance, largely the management of number of factors including: financial, time, data and people management. A development framework requires to be established within the enterprise to overcome these barriers. The results from the case study analysis showed $75 \%$ of participants will improve time efficiencies as a result of learning new IT. The proposed solutions of the bespoke IT management solution highlight the potential of a significant timeefficiency improvement on administrative tasks using IT in a microenterprise. The study aims to facilitate future design of digital handbook for training and performance management of employees for the company. This research suggests greater potential with the right balance of suitable solutions, time and skills adopted to address this question, hence the solutions offers the potential to address this.

\section{REFERENCES}

[1] G. Johnson, K. Scholes, and R. Whittington, "Exploring Corporate Strategy, " $8^{\text {th }}$ edition Harlow: Pearson Education Limited, 2008.

[2] R. Tyler, "Explosion of micro companies recorded", Telegraph New Article on finance and your business, 2015.

[3] European Union., "Offical Journal of the European Union", vol. 46, pp.36, 2003.

[4] D. Devins, S. Johnson, J. Gold, and R. Holden, "Management Development and Learning in Micro Businesses: a 'missing link' in research and policy," Report prepared for: Research and Evaluation Unit Small Business Service. Leeds: Leeds Metropolitan University Policy Research Institure, 2001.

[5] J.S. Walters, "Big Vision, Small Business: 4 Keys to success without growing big," 7th edition, San Fransisco: Berrett-Koehler Publishers Inc, 2002.

[6] Department of Business Innovation and Skills (BIS), "BIS Small Business Survey Report” Department for Business Innovation \& Skills; [Online] Available at: $<$ www.gov.uk/government/uploads/system/uploads/attach ment_data/file/32228/11-p74-bis-small-business-survey2010.pdf $>$ [Accessed 10 May 2017], 2010.

[7] F. Lasch, F. Le Roy, S. Yami, "Critical growth factors of ICT start-ups," Management Decision, vol. 45, no. 1, pp.62-75, 2007.

[8] C.H. Davis, C. Lin, and F. Vladica, "Internet Technologies and e-business solutions among small and medium-sized enterprises (SMEs) in Atlantic Canada - Patterns of Use, Business Impacts, and demand for Support Services," In Proceedings of the 7th World Congress on Management for e-Business. Halifax, 2006.

[9] Federation of Small Business (FSB), "The FSB 'Voice of Small Business' Member Survey". Birmingham: Federation of Small Business, [Online] Available at: www.fsb.org.uk/docs/default-source/fsb-org-uk/policy/ [Accessed 12 May 2017], 2013.

[10] A. Berry, and L. Perren, "The role of non-executive directors in UK SMEs," Journal of Small Business and Enterprise Development, vol. 8, no. 2, pp.215-32, 2001.

[11] R.T. Rust, F. Espinoza, "How Technology advances influence business research and marketing strategy," Journal of Business Research, vol. 59, pp.1072-78, 2006.

[12] M. Jouvernaux, Micro-enterprises, technology and ECommerce in New Zealand., In 11th Pacific-Asia Conference on Information Systems. Auckland, 2007. PACIS.

[13] P. Wolcott, M. Kamal, and S. Qureshi, "Meeting the challenges of ICT adoption by micro-enterprises," Journal of Enterprise Information Managment, vol. 21, no. 6, pp.616-632, 2008.

[14] P. Evangelista, A. McKinnon, and E. Sweeney, Technology adoption in small and medium-sized logistics providers. Industrial Management \& Data Systems, vol. 113, no. 7, pp.967-989, 2013.

[15] S. Shah, and S. Hasan, "Influence of IT on Micro Enterprises to pursue Strategic Growth," Proceedings of the 19th International Conference on Computers - CSCC 2015, vol. 32, pp.488-496, 2015. 